Instituto Internacional de Investigación y Desarrollo Tecnológico Educativo INDTEC, C.A.

DOI: https://doi.org/10.29394/scientific.issn.2542-2987.2017.2.3.1.11-30

OAI-PMH: http://www.indteca.com/ojs/index.php/Revista Scientific/oai

\title{
Comunidad de Aprendizaje, la Reciprocidad de Aprender y Enseñar. Tú me Enseñas, yo te Enseño y todos Aprendemos
}

Autores: Gladys Isabel Portilla Faicán Universidad Nacional de Educación, UNAE gladys.portilla@unae.edu.ec Azogues, Ecuador Mario Rosendo Dután Cuenca Universidad Nacional de Educación, UNAE mario.dutan@unae.edu.ec Azogues, Ecuador Hugo Fernando Abril Piedra Universidad Nacional de Educación, UNAE hugo.abril@unae.edu.ec Azogues, Ecuador Jaime Iván Ullauri Ullauri Universidad Nacional de Educación, UNAE jaime.ullauri@unae.edu.ec

Azogues, Ecuador

\section{Resumen}

El siguiente trabajo narra la experiencia de la formación y desarrollo de una comunidad de aprendizaje docente en la Universidad Nacional de Educación (UNAE de Ecuador). La iniciativa surgió de un grupo de docentes ante la necesidad de desarrollar competencias digitales para innovar su práctica docente. Se recurrió a la comunidad de aprendizaje, por ser considerado un espacio caracterizado por la familiaridad y horizontalidad de las interrelaciones entre pares para la construcción de aprendizajes colaborativos. La propuesta se enmarca en el conectivismo como una versión actual del constructivismo en el contexto de la era digital. A través de la metodología de talleres se exploró el uso de recursos digitales en función de necesidades, contextos disciplinares y metodológicos específicos del docente universitario en esta era. Se vivenció el rol e impacto de las tecnologías para el aprendizaje y el conocimiento (TAC) por medio del abordaje de recursos como: Academic Writing, Wikis y Online presentation, considerados de interés en la comunidad de aprendizaje. Esta experiencia ha desembocado en resultados contundentes que corroboran la efectividad e impacto positivo de la comunidad de aprendizaje para afrontar los desafíos de la era digital.

Palabras clave: aprendizaje colaborativo; comunidades de aprendizaje; innovación educativa; docente.

Fecha de Recepción: 15-08-2016 Fecha de Aceptación: 19-09-2016 


\title{
Learning Community, Reciprocity of Learning and Teaching. You Teach me, i Teach and Learn all
}

\begin{abstract}
The following work narrates the experience of the formation and development of a community of teacher learning in Universidad Nacional de Educación (UNAE de Ecuador). The initiative emerged of teachers group to face the necessity to development digital competences in order to innovate their teaching practices. It was resorted to the learning community, being considered a space characterized by familiarity and horizontality of the interrelationships between pairs for building collaborative learning. The proposal is part of the connectivism as a current version of constructivism in the context of the digital age. Through workshops methodology using digital resources according to needs, specific disciplinary and methodological contexts of university teachers in this era was explored. It was evident the role and impact of learning technologies and knowledge (TAC) through addressing resources: Academic Writing, Wikis and Online presentation considered of interest in the learning community. This experience has led to strong results confirm the effectiveness and positive impact of learning community to face the challenges of the digital age.
\end{abstract}

Keywords: collaborative learning; learning communities; educational innovation; professor.

Date Received: 15-08-2016

Date Acceptance: 19-09-2016 
"Todos los días enseñas algo, todos los días aprendes algo"

\section{Introducción}

Uno de los desafíos de la docencia en la actualidad es la innovación y pertinencia de la práctica docente en la era digital. Los niños y jóvenes habitan gran parte de su tiempo en entornos digitales, sin embargo, en las aulas y otros espacios de aprendizaje formal no pueden usar las competencias y saberes del mundo digital, debido principalmente a que los docentes no están familiarizados con los entornos virtuales. El modelo pedagógico y curricular de la Universidad Nacional de Educación (UNAE), que se caracteriza por la innovación y contextualización en la era digital, supone el uso de tecnología educativa digital, como un eje transversal de los procesos de enseñanzaaprendizaje (Vilanova, 2015). Al describir una de las competencias profesionales del docente contemporáneo, se hace referencia a que "los contextos hoy son inevitablemente, querámoslo o no, virtuales y presenciales. No podemos concebir una escuela del siglo XXI exclusivamente con los espacios presenciales, necesitamos compartir, integrar y potenciar los espacios virtuales" (Comisión Gestora de la Unae, 2015. pág. 19), Alfredo Hernando (2015) coincide en que la educación en este siglo debe incorporar la dimensión virtual en el aula.

El proceso educativo que actualmente se desarrolla en las aulas de clases de las instituciones educativas, es permeado por el mundo virtual y tecnológico desde diversas aristas, en los procesos técnico-administrativos, los espacios de formación docente, las actividades inherentes al ejercicio docente en cuanto a las planificaciones académicas, las estrategias pedagógicas-didácticas y actividades de evaluación de los aprendizajes (Peralta, 2016). En este sentido, la UNAE posee el compromiso con la sociedad ecuatoriana de formar los futuros docentes desde y para los 
ambientes de aprendizaje virtuales, actores que han de agilizar la instauración de un modelo pedagógico con un componente de tecnología virtual en los diversos niveles y modalidades del sistema educativo, equilibrando los escenarios de aprendizaje para facilitar el transitar desde modelos pedagógicos tradicionales a escenarios de aprendizaje equilibrados ante los requerimientos de la educación en la era digital.

Por tal motivo en una reunión de docentes coordinadores de asignatura, en Nivelación de Carrera se planteó la necesidad de crear espacios de formación docente para el uso de tecnología digital, con el fin de afrontar los desafíos de la docencia, de cara a los intereses y hábitos de los estudiantes y llevar a la práctica docente cotidiana los principios pedagógicos del Modelo UNAE. Ante esta necesidad, desde la Coordinación Académica de Nivelación se tomó la iniciativa de formar un equipo de gestión de espacios de desarrollo profesional docente, bajo los principios filosófico-pedagógicos de la UNAE. EI objetivo fue formar una comunidad de aprendizaje con el propósito de afianzar y desarrollar las competencias digitales del docente UNAE, a través de espacios de socialización de saberes y habilidades, en talleres de desarrollo de tecnologías para el aprendizaje y el conocimiento (TAC). Una de las competencias profesionales del docente contemporáneo es justamente la "Capacidad para diseñar y construir contextos y comunidades de aprendizaje" (Comisión Gestora de la UNAE, 2015. pág. 19). En esta era como en ninguna otra los aprendizajes se desarrollan de forma colectiva, y explorar la tecnología educativa digital y su mundo virtual es una cuestión comunitaria.

Se diseñó la propuesta desde la lógica de una comunidad de aprendizaje, en la que es concebida por el equipo de gestión, como un espacio alternativo para la socialización de dudas, preocupaciones, necesidades, intereses, conocimientos y saberes. Estos espacios están pensados para responder a la pregunta básica y estructuradora de itinerarios a seguir: ¿Qué queremos aprender? La idea es aprender 'con' y 'de' otros, en un ambiente de 
horizontalidad, propio de las interrelaciones de pares. La novedad de la propuesta es pasar de los roles de facilitador y facilitado o participante a la hibridación de roles. Hay un equipo de gestión del proceso, pero ya en los talleres, que fue la metodología adoptada, los miembros del equipo de gestión presentan la temática y en su desarrollo todos aprenden y enseñan algo. No hay el rol de experto, pues se trata de aprovechar al máximo al carácter intuitivo del mundo virtual. Bajo estas concepciones se decidió conformar la Comunidad de aprendizaje, la reciprocidad de aprender y enseñar, en torno a la tecnología educativa emergente.

Se hizo la invitación a los docentes de la comunidad universitaria, al mismo tiempo que se consultó sobre sus intereses de formación, desarrollo profesional y niveles de dominio sobre 18 categorías de recursos educativos digitales, con sus principales herramientas y usos educativos, vinculados con la Web 2.0, considerada la tecnología educativa de vanguardia. Para el desarrollo de un primer ciclo de talleres se tomó como temas las tres categorías más votadas como de interés para aprender a usarlas: Academic Writing, Wikis y Online presentation. El proceso y los resultados del primer ciclo de talleres desarrollados en diferentes jornadas y con una duración de ocho horas fueron evaluados por los participantes, mayoritariamente como excelente y en algunos casos como muy buenos. Los trece participantes manifestaron su interés en volver a participar en una comunidad de aprendizaje y recomendaron continuar con esta práctica.

\section{Metodología}

Se formó el equipo de gestión de la comunidad de aprendizaje de docentes en la UNAE con cuatro miembros. Se procedió al planteamiento de objetivos operativos: a). Indagar los intereses y necesidades de formación de los docentes. b). Diseñar la metodología y actividades iniciales de la comunidad. c). Diseñar las estrategias e instrumentos de evaluación de la 
primera fase de la comunidad de aprendizaje. Previa consulta a las instancias directivas pertinentes, se envió a los correos electrónicos de los docentes la invitación, a la que se adjuntó una tabla de usos, categorías y recursos, de los cuales se pidió seleccionar las categorías con las que más les interesaba familiarizarse; también aquellas con las que más estén familiarizados. Las categorías propuestas fueron: 1. Blogging, 2. Chat Tools, 3. Charts \& Graphs, 4. File Sharing \& Mashups, 5. Microblogging, 6. Notebooks \& Annotation Tools, 7. Online Presentation, 8. Personal Web Portals, 9. Photo Editing/Sharing, 10. Search Engines, 11. Social Bookmarking, 12. Social Network, 13. Survey \& Polling Tools, 14. Task \& Group Management, 15. Video Chat, 16. Video Editing/Sharing, 17. Wikis, 18. Academic Writing. Las tres que más interés suscitaron fueron: Academic Writing, Wikis y Online presentation. La mayoría de las categorías resultaron desconocidas. En la lógica del aula invertida, en los días previos a cada taller se les enviaba a los docentes videos cortos, en los que se presentaba usos y conceptos básicos de cada herramienta, de tal forma que en el taller se aproveche al máximo para el uso práctico de las mismas.

La invitación tuvo el carácter de voluntaria y según intereses, por lo tanto, participaron trece docentes, quienes estaban interesados y en función de su tiempo libre. Luego, considerando las categorías elegidas, se organizaron los talleres en salones de clase y con el uso de los computadores personales de cada docente. Se prescindió del uso de un laboratorio de informática, con el fin de crear las condiciones de un entorno físico familiar y para facilitar la movilidad de un lugar a otro, para pedir o dar ayuda a un compañero, para observar, tocar la pantalla o el mouse del computador de otro compañero. La idea fue diseñar el taller dentro de un contexto de aprendizaje colaborativo, en el que predomine el principio de aprender 'con' y 'de' otros, entre todos. Los docentes compartieron en los talleres experiencias, expectativas, preguntas, dudas, tips, entre otros. 
A través de la observación participante se registraron los eventos y relaciones interpersonales que se dieron en torno a la exploración y uso de los recursos digitales: ¿cómo se abordaron y resolvieron dudas?, ¿cuál fue la actitud al pedir y dar ayuda para superar dificultades puntuales?, los comentarios hechos sobre los recursos digitales y el taller, en general; es decir, la construcción de aprendizajes en una comunidad de docentes aprendientes. En los talleres se exploró el uso de recursos digitales en función de necesidades y contextos disciplinares y metodológicos específicos, evidenciando el rol de las TAC. A su vez se hizo una evaluación de nociones y habilidades básicas desarrolladas usando la herramienta Kahoot. Finalmente se evaluó la propuesta, desde la convocatoria a participar en la misma hasta su proceso de desarrollo y resultados, a través de una encuesta en Survey Monkey.

\section{Resultados y Discusión}

\subsection{El desafío para la docencia en la era digital}

A continuación, se presenta una breve compilación de aquellos principios educativos del modelo pedagógico de la UNAE, que guardan estrecha relación con el tema de la tecnología educativa digital, en virtud de que en su estructura curricular el 30\% del programa de formación docente debe destinarse a la utilización de plataformas virtuales para el desarrollo de actividades pedagógicas diversas en el desarrollo de los contenidos de los aprendizajes significativos.

La era digital requiere aprendizajes de orden superior que ayuden a vivir en la incertidumbre y la complejidad. Los estudiantes deben aprender en conectividad, en Entornos Virtuales de Aprendizaje (EVA), por ello es fundamental desarrollar y estimular la función tutorial del docente. El Conectivismo tal como lo propone Siemens (Rodríguez, 2012), supone la versión actual del Constructivismo al tomar en consideración el contexto digital 
ilimitado de los intercambios humanos. Destaca que los escenarios de aprendizaje en los que se encuentra el conocimiento distribuido y al alcance de todos, ofreciendo oportunidades insospechadas de aprendizaje, son las inabarcables redes telemáticas de acumulación, intercambio y creación permanente de información y conocimiento. En cuanto al contexto normativo educativo el Reglamento de Régimen Académico, en los artículos 15, 28 y 38, señala que las actividades de aprendizaje deben incluir el uso adecuado de diversas tecnologías de la información y la comunicación, así como actividades en red, tutorías en entornos virtuales, lo que implica habilidades para la comunicación y lenguajes digitales. En el Plan Nacional del Buen Vivir 20132017 (Senplades, 2013), al referirse a la productividad y competitividad, se sostiene que en un contexto a largo plazo se consolidarán los territorios y las ciudades digitales, que se caracterizarán por el uso de las TIC. En conclusión, el aprendiz vive el intercambio con la información digital y las redes sociales en el escenario y en la atmósfera que rodea su vida fuera del aula (Comisión Gestora de la UNAE, 2015). Este es el contexto del mundo social de niños y jóvenes, donde ocurren gran parte de los aprendizajes no formales, situación de gran relevancia para los aprendizajes escolarizados, y por lo tanto para los docentes. En pocas palabras, según Ángel Pérez G., hay nuevas demandas educativas (2012).

Los entornos virtuales y sus recursos asociados se han convertido en un tema central en la innovación educativa, y por ello ocupan un lugar desatacado en el Modelo Pedagógico de la UNAE. El Conectivismo es uno de los componentes medulares de un modelo. Una de las competencias básicas del docente UNAE es "Promover la didáctica invertida, la flipped classroom, las redes sociales virtuales y las plataformas digitales" (Comisión Gestora de la Unae, 2015. pág. 8). En esta didáctica el rol protagónico lo desempeñan estudiantes habituados a navegar en la web, leer hipertextos y en hiperpantallas. En este escenario, uno de los principios pedagógicas es: 
"aprovechar al máximo los recursos digitales y las redes sociales, plataformas, laboratorios, museos, enciclopedias y talleres virtuales, herramientas de diseño digital, espacios virtuales de cooperación, plataformas e instrumentos multimedia, con el propósito de fomentar la competencia digital como usuarios activos y creativos de todos los aprendices" (Comisión Gestora de la UNAE, 2015. pág. 20).

En la actualidad la cultura digital está poblando todos los espacios en los que se habita y ello involucra de manera necesaria y urgente a quienes tienen que ver con el mundo de la docencia.

Los docentes debemos afrontar de forma proactiva y creativa los desafíos de enseñar en la era digital (Yigit, 2013). En este espacio de formación se procura alcanzar un nivel de análisis desde la perspectiva de la mediación pedagógica de la alfabetización digital. Desde la perspectiva conectivista de George Siemens el aprendizaje se produce en redes y ecosistemas de conocimiento (Rodríguez, 2012). Por su parte Freddy Álvarez propone cinco principios del Buen Vivir para la educación y uno de ellos es la comunitariedad versus la individualidad (2015). En este contexto emerge la propuesta de la Comunidad de aprendizaje, la reciprocidad de aprender y enseñar.

\subsection{La experiencia del aprendizaje cooperativo entre docentes}

A pesar de que el trabajo colaborativo está en boga en los ámbitos docentes; sin embargo, en sus procesos de formación continua se da mucho crédito a la capacitación en cursos dictados por expertos. La propuesta de comunidad de aprendizaje trata de superar la dicotomía y verticalidad de roles que implica este tipo de formación: el de enseñante y el de aprendiente. Esta experiencia partió de la concepción de hibridación de roles, en la que se enseña y aprende, de forma simultáneo o alternada. Lakomski habla de valorar las habilidades colectivas (Lakomski, 2011). Todos traen saberes previos que 
aportan al aprendizaje de los miembros de la comunidad, en la misma se logran aprendizajes que al mismo tiempo que suceden e inmediatamente se comparten y multiplican. Se evidenció que los docentes, más que cualquier otro profesional, aprenden enseñando. Paulo Freire dice que la tarea del docente es también la de aprendiz (2010). En los talleres desarrollados, desde el primer día se observó que afianzaban lo logrado al compartir con el compañero de lado, o cuando alguien pedía ayuda para algo y hubo quienes se daban cuenta que ellos ya lo lograron, otros que también tenían el mismo problema y aprovechaban la explicación. Esto permitió que se tejiera un entramado de ayuda mutua entre los participantes. De este modo se vivió el intercambio de roles. Una frase dicha por una compañera refleja esta dinámica: "Tú me enseñas, yo te enseño y todos aprendemos".

Se aprendió haciendo. Se vivió la experiencia de uno de los principios de la fundamentación psicológica del modelo pedagógico UNAE, el enactivismo: "conocer en la acción, incluyendo la interacción corporal, experiencial y cognitiva. No solo se habla de cognición situada sino principalmente de acción situada en un contexto percibido como singular" (Comisión Gestora de la UNAE, 2015. pág. 10). La comprensión del uso de las herramientas emergía de lo que hacían cada uno y en pares o grupos, lo cual se iba reflejando en la pantalla del proyector y en la de sus ordenadores personales. Al explorar con OneDrive la escritura colaborativa online y observar la interactividad y colaboración que se puede lograr con este recurso multimedia, los docentes se veían a sí mismos y los demás editando el texto, insertando recursos multimedia, referenciando desde Word y con Mendeley, ésta última fue instalada por los mismos docentes en los computadores personales. Otro recurso que se exploró fue Prezi. Los docentes crearon sus cuentas e hicieron su primera presentación online, explorando sus posibilidades básicas, se puso énfasis en aprovechar su carácter dinámico y multimedia. 
Hubo momentos de confusión y de logro, de pregunta y respuesta sobre la marcha, en situaciones puntuales. Cuando alguien no entendía algo o había perdido la secuencia de algún tema pedía ayuda inmediatamente al compañero de lado o a quienes coordinaban el taller, se volvía sobre puntos de interés para algunos; en una lógica de resolver sobre la marcha los problemas presentados y en función de las necesidades individuales y puntuales. En estos momentos se afianzaban algunos conocimientos recién alcanzados al ayudar a alguien que tenía dificultades.

Entre las principales ventajas enfatizadas por los docentes, en torno al uso de estos recursos virtuales, fue la optimización del tiempo y espacio, posibilidad de compartir en línea, la real posibilidad de desarrollar la escritura colaborativa y la retroalimentación de los textos de estudiantes y pares, a través del comentario o la inclusión de recursos multimedia en el texto, la simplificación de la referencia bibliográfica y lo dinámico y didáctico de Prezi.

\subsection{Excelente, hay que seguir haciéndolo}

Los docentes participantes de la Comunidad de aprendizaje, la reciprocidad de aprender y enseñar, evaluaron esta primera experiencia a partir de preguntas tales como: ¿Ha participado antes de una comunidad de aprendizaje? La mayoría respondió que no. Entre las principales motivaciones para participar en la comunidad de aprendizaje se dijo que fue el interés por innovar su práctica docente y la necesidad de manejar las herramientas tecnológicas propuestas. Todos dijeron haber aprendido algo en esos talleres. Entre lo más interesante que dijeron haber aprendido está en primer lugar la referenciación bibliográfica con Mendeley y desde Word, luego Prezi y finalmente Kahoot (esta herramienta se usó para evaluar, pero los docentes también consideraron como algo interesante que han aprendido para trabajarlo en sus aulas). A la pregunta ¿Cómo aprendió?, respondieron que con la ayuda de los compañeros, a través del ensayo y error, con explicaciones puntuales y 
con la práctica continua y en la interrelación grupal. Aquí algunas expresiones textuales: "Lo más interesante de las sesiones en la comunidad fue el intercambio, todos aprendíamos de todos y todos nos enseñábamos. Los facilitadores y tutores estuvieron siempre dispuestos a ayudarnos y lo hacían desde la horizontalidad; algo muy valioso.", "Desde la interacción con mis compañeros y tutores", "De forma práctica y colaborativa".

A la pregunta de ¿Cómo está usando o usaría los recursos socializados en estos talleres? Respondieron textualmente: "Mis próximas presentaciones en el aula serán en Prezi. Para los artículos de investigación se me hace más fácil colocar las referencias en Mendeley y citar por Word", "En mi doctorado y proyectos con mis estudiantes", "En el aula, y con compañeros investigadores", "Los usaría en mi práctica diaria para una mejor instrucción interactiva y efectiva", "Obviamente en el aula con los estudiantes, en el desarrollo de nuestros artículos e investigación", "En primer lugar para mi clase $y$, en segundo lugar, para la elaboración de artículos, proyectos". Esto da cuenta de la utilidad y practicidad de los recursos y el nivel de conocimiento y dominio alcanzados.

Más del $90 \%$ dijo haber ayudado a alguien a aprender, sólo uno respondió que no. Lo que pone en evidencia que una comunidad de aprendizaje es el espacio propicio para que todos ayuden a aprender a los demás sin ser expertos, ni desde el rol de facilitadores, sino desde el aprendizaje colaborativo, que tiene un carácter multiplicador; por ejemplo, cuando alguien lograba insertar una referencia bibliográfica con Mendeley, los que están a su alrededor lo hacían con su ayuda. Los compañeros del equipo de gestión han sido quienes tienen cierta experticia en el uso de los recursos explorados, pero la idea, desde la concepción misma de la comunidad de aprendizaje, fue minimizar el rol de facilitadores y potenciar el de aprendientes colaborativos. Se procuró siempre desdibujar los tradicionales roles de facilitador y facilitado, lo que se ha logrado en gran medida, sin embargo, aún 
se percibe la presencia de estos roles en los criterios de los docentes al referirse a los miembros del equipo de gestión como los facilitadores.

Con respecto a la presentación de la propuesta y la convocatoria a los talleres (el formulario de preferencias y los videos de introducción a las temáticas de los talleres), un $73 \%$ calificó de excelente y el $23 \%$ de muy bueno. La metodología para el desarrollo de los talleres (hacer juntos, equipo de gestión y participantes, la exploración y uso de los recursos, preguntar y responder preguntas según vayan surgiendo) fue considerada excelente por un $73 \%$ y de muy buena por el $23 \%$. El ambiente de las interrelaciones personales en el transcurso de los talleres fue calificado como excelente por un $91 \%$ y muy bueno por un $9 \%$. El $100 \%$ calificó de excelente la utilidad de los aprendizajes desarrollados. Lo novedoso e innovador de esta forma de construir conocimientos, en un ambiente comunitario y entre pares fue evaluado como excelente por un $91 \%$ y muy bueno por un $9 \%$. La innovación de los recursos abordados fue considerada excelente por un $73 \%$ y de muy buena por el $23 \%$.

Se preguntó, con base en esta experiencia, ¿cómo definiría una "comunidad de aprendizaje"? Estas son algunas respuestas textuales: "Trabajo colaborativo" "Todos aprendemos de todos", "Espacio eficaz e interactivo para aprender", "Grupo de personas motivas por generar nuevos conocimientos conjuntamente", "Aprendizaje colaborativo", "Todos aprendemos de todos", "Excelente. Muy necesaria y útil”, "Trabajo colaborativo los estudiantes se convirtieron en docentes, cada uno aportó desde su experiencia y conocimiento", "Es un espacio de comunicación en la que todos aprendemos y enseñamos a otros sobre diversos tópicos de interés para todo el grupo", "Como un espacio idóneo para la construcción de aprendizajes significativos colaborativos". Estas definiciones de los miembros de la comunidad de aprendizaje guardan estrecha relación con la concepción de comunidad de aprendizaje de la que partió la propuesta; un espacio de 
encuentro de intereses y preocupaciones comunes en torno a las que se aprende 'con' y 'de' otros, en un ambiente de horizontalidad.

Las recomendaciones que se hicieron fueron las siguientes: "más frecuencia y nuevos temas", "Realizarlo con más tiempo para más práctica y mejor comprensión", "Mejor conectividad", "Recomendaría enviar tareas a casa y retroalimentarla en línea", "Recomiendo que se repita la experiencia y que se agreguen más temas y más personas. ¡Felicito a los organizadores y facilitadores de esta extraordinaria experiencia de aprendizaje ya que no solo aprendimos desde el máximo placer y motivación, sino que estrechamos lazos de compañerismo y amistad!!” ¡Excelente iniciativa!! Felicitaciones Mario, Hugo, Gladys, Jaime..." "Hay que seguir haciéndolo". En un segundo ciclo de talleres se tendrá en consideración las recomendaciones.

El tiempo fue un factor muy importante en el número de participantes y en el cumplimiento de la planificación. Al haber desarrollado la propuesta a final de semestre, algunos docentes manifestaron no poder participar, a pesar de su interés. Por otro lado, el tiempo previsto no fue suficiente para desarrollar las tres temáticas planificadas, no se abordó la temática de las Wikis. Además, los docentes recomendaron más tiempo para consolidar los aprendizajes.

\section{Conclusiones}

Los profesores, más que cualquier otro profesional, aprenden enseñando, afianzan lo logrado al compartir con otros. Esta experiencia permitió explorar la potencialidades y ventajas del intercambio de roles, en una dinámica continúa llegando a la hibridación de roles. Esta experiencia demostró que se aprende más y mejor cuando se enseña y se aprende, que cuando sólo se aprende o enseña.

La tecnología educativa digital es un desafío que es más fácil afrontarlo con éxito si se lo hace con otros, en comunidades. El carácter altamente intuitivo de los recursos digitales, más la posibilidad de sumar y multiplicar 
aprendizajes individuales es una excelente estrategia para innovar la práctica docente en el escenario de la era digital. Estos talleres, en torno a la escritura académica colaborativa y presentaciones online, demostraron importantes ventajas y posibilidades de optimizar tiempo y recursos, por un lado y por otro, la posibilidad real de pasar a una escritura académica colaborativa online, muy didáctica y eficaz. Los recursos digitales explorados fueron apreciados como potentes estrategias de innovación de la escritura académica, como tarea sustancial del mundo académico y la docencia, en específico.

Esta experiencia podría convertirse en la fase inicial de una cultura profesional de la innovación docente y desarrollo profesional continúo basada en el aprendizaje en comunidad. La propuesta logró hacer de ese espacio de formación una vivencia compartida del lema de la UNAE: "Todos los días enseñas algo, todos los días aprendes algo." El carácter opcional y voluntario de la invitación y permanencia en la comunidad fue un factor de compromiso y motivación que influyó en los excelentes resultados alcanzados en el primer ciclo de talleres de la Comunidad de aprendizaje, la reciprocidad de aprender y enseñar.

\section{Referencias}

Alvarez, F. (2015). Universidad emblemática de maestros y maestras para el Buen Vivir. Un acercamiento a una propuesta de identidad. Hacer bien, pensar bien y sentir bien., pp. 1-11. Azogues.

Comisión Gestora de la UNAE. (2015). Modelo pedagógico de la UNAE, nro.

28. Ecuador: Universidad Nacional de Educación.

Freire, P. (2010). Cartas a quien pretende enseñar. Siglo Veintiuno Editores S.A., Ed. Buenos Aires.

Hernando, A. (2015). Viaje a la escuela del siglo XXI. Madrid. 
Lakomski, G. (2011). Saber cómo aprender: liderazgo, gestión del conocimiento y el reto de crear comunidades de aprendizaje. Educar, 47(47), 13-30. Recuperado de:

http://ddd.uab.cat/pub/educar/0211819Xv47n1/0211819Xv47n1p13.pdf

Peralta, L. (2016). La computadora en el aula, ayuda o complicación con el maestro. Mamakuna, 2, 46-55.

Pérez, Á. (2012). Educarse en la era digital. Morata.

Rodríguez, P. (2012). Conectivismo: George Siemens y el aprendizaje en red. Un paso más. [Documento en línea]. Recuperado de: https://unpasomas.fundaciontelefonica.com/2012/10/18/conectivismogeorge-siemens-y-el-aprendizaje-en-red/

Senplades (2013). Plan Nacional Buen Vivir. 2013-2017. SENPLADES. Quito.

Vilanova, M. (2015). Coordinación Académica. Hacer bien, pensar bien y sentir bien., pp. 79-82. Azogues: UNAE.

Yiğit, Ö. (2013). Science, Technology and Social Change Course' S Effects on Technological Literacy Levels of Social Studies Pre-Service. TOJET: The Turkish Online Journal of Educational Technology, 12(3), 142-156. 


\section{Gladys Isabel Portilla Faicán}

e-mail: gladys.portilla@unae.edu.ec

Formación de tercer nivel y cuarto nivel en Ciencias de la

Educación y Ciencias Sociales. En la actualidad doctorante en la Universidad de La Habana. Profesora de la Universidad Nacional de Educación (UNAE-Ecuador). Docente-Investigadora en las áreas de Ciencias sociales, Didáctica de la Ciencias sociales, Formación docente, Innovación educativa y Tecnología educativa emergente. 


\section{Mario Rosendo Dután Cuenca}

e-mail: mario.dutan@unae.edu.ec

Licenciado en Psicología Educativa, Universidad de Cuenca - Ecuador. Coordinador de Nivelación y Admisión, Universidad Nacional de Educación UNAE. Coordinador Zonal y Facilitador del Programa de Formación Continua "Sí Profe", Ministerio de Educación del Ecuador. Coordinador Zonal del proyecto Territorios de Paz del Centro de Arbitraje y Medicación. Facilitador del Plan Estratégico Institucional para la Dirección de Educación y Cultura (GADMA). Investigador, elaboración de línea base del estado de cumplimiento de los derechos de los niños, niñas y adolescentes del cantón Nabón-Azuay. Técnico Nacional de Desarrollo Infantil. MIES.

Coordinador Nacional de Justicia y Cultura de Paz del Consejo de la Judicatura. 


\section{Hugo Fernando Abril Piedra}

e-mail: hugo.abril@unae.edu.ec

Cuenca, Ecuador, 1971. Magíster en Gerencia y

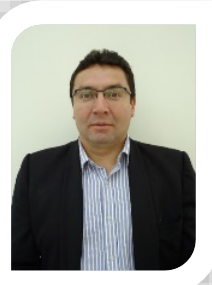

Liderazgo Educativo por la Universidad Técnica

Particular de Loja, Ingeniero de Sistemas y Analista de

Sistema otorgado por la Universidad Católica de Cuenca.

Desde 1995, ha estado vinculado a Instituciones que pertenecen al Sistema de Educación Superior del Ecuador de nivel de pregrado y de formación técnica y tecnológica, en calidad de Docente y Directivo. Su objetivo como profesional en el área de la Educación es relacionar la tecnología en el proceso de enseñanzaaprendizaje, lo que le ha llevado a participar en los Colectivos Académicos de Investigación de la UNAE. 
Jaime Iván Ullauri Ullauri

e-mail: jaime.ullauri@unae.edu.ec

Licenciado en Psicología Educativa y Magíster en

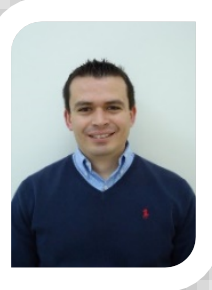

Educación y Desarrollo del Pensamiento por Universidad

De Cuenca - Ecuador. Máster en Orientación Educativa

por Universidad Nacional de Educación Distancia-

España. Docente-Investigador en la catedra de Aprendizaje Humano y docente de Nivelación General en Universidad Nacional de Educación, ha participado en procesos similares en la Universidad de Cuenca. Docente de Educación General Básica y bachillerato dentro del área de Estudios Sociales y Desarrollo del Pensamiento Filosófico. Facilitador Académico del Programa de Asesoría y Auditoría Educativa y del Programa de Formación Continua "Sí Profe" del Ministerio de Educación del Ecuador.

El contenido de este manuscrito se difunde bajo una Licencia de Creative Commons ReconocimientoNoComercial-Compartirlgual 4.0 Internacional 